\title{
The misleading use of the term pragmatic in pre-licensing medicine trials
}

\author{
Rafael Dal-Ré ${ }^{1}$ \\ Received: 8 March 2019 / Accepted: 20 March 2019 /Published online: 26 March 2019 \\ (C) Springer-Verlag GmbH Germany, part of Springer Nature 2019
}

Randomized controlled trials (RCTs) could be designed and conducted with two completely different approaches: explanatory (ideal controlled conditions, strict selection criteria, homogeneous population, aimed to assess efficacy) or pragmatic (clinical practice conditions, broad selection criteria, heterogeneous population, aimed to assess effectiveness). Yet, these are the extreme of a continuum. Most RCTs could have both explanatory and pragmatic features. The PRECIS-2 tool was designed to assess how much pragmatic (or explanatory) is a trial by carefully assessing nine domains - from the eligibility criteria and recruitment method to the follow-up and statistical analysis [1]. When sponsors of RCTs tagged them as "pragmatic," they want to convey the message that the trials were conducted resembling real-world clinical practice yielding generalizability results [2] and, hence, have special value for patients, prescribers, and policymakers. The degree of pragmatism of RCTs can vary a lot, from being highly pragmatic, with several PRECIS-2 domain scores in the pragmatic extreme, to having most features close to the explanatory extreme [2].

But, when does a trial deserve to be labelled as pragmatic? It has been shown that in the majority of cases, trialists did not justify the pragmatic tag or believed that their trial was pragmatic because it fulfilled one of the nine PRECIS-2 domains [3]. However, if we accept this approach as correct, how can we label those trials that had a pragmatic approach on, for instance, six or seven PRECIS2 domains? While the clinical trials community tries to agree on this, both industry and some academic investigators are (incorrectly) using the adjective "pragmatic" to label pre-licensing (pre-approval) medicine trials, trials

Rafael Dal-Ré

rafael.dalre@quironsalud.es

1 Health Research Institute-Fundación Jiménez Díaz University Hospital, Universidad Autónoma de Madrid, Avda Reyes Católicos 2, E-28040 Madrid, Spain conducted to assess the efficacy and safety of new indications or of investigational medicines. Two recently published RCTs in the top two internal medicine journals, conducted in the UK, exemplify the opposite extreme approaches taken by industry and academics when deciding to label as pragmatic a pre-licensing medicine RCT.

The first RCT, the Salford COPD trial, was published in 2016. This was an industry-sponsored multicenter trial to compare an investigational medication (fluticasone furoate and vilanterol) vs usual care in chronic obstructive pulmonary disease (COPD) [4]. This study was labeled as "the world's first phase 3 pragmatic RCT" (emphasis added) in COPD patients. Since 2012 on the registry (clinicaltrials.gov) and in all published reports since 2014, the terms "pragmatic," "realworld," "effectiveness" (as opposed to efficacy [5]), or "normal clinical practice" were repeatedly mentioned [6]. That this was a phase 3 trial was also mentioned in all reports, except in the article reporting the results. The assessment of this trial by means of the PRECIS-2 tool nine domains showed that the Salford COPD trial had few domains in the pragmatic extreme and others in the explanatory extreme [6]. Although this was an open-label RCT, it was conducted following the EU clinical trials regulation that mandates a number of procedures and processes that distorted the usual patient-physician encounter and the follow-up of participants. The fact that, as has been claimed, there were few exclusions and that some $50 \%$ of eligible patients took part in the trial [7] is far from being enough to consider a trial as pragmatic [2]. In addition, it has been recently described that both physicians and patients were subject to behavioral changes due to the Hawthorne effect [8] - something that should be expected to happen to some extent in almost all trials. So, this industry-sponsored phase 3 trial was labelled as "pragmatic" since its inception, but the term was omitted in the most important paper, the one reporting the results-although other terms intimately associated to "pragmatic" trials such as "real-world" and "usual" care were repeatedly mentioned [4]. 
In the opposite extreme was the approach taken by the authors of FOCUS that tagged as pragmatic their doubleblind, placebo-controlled trial [9]. This was a multicenter RCT comparing fluoxetine with placebo on functional outcomes after acute stroke published in 2019. Hence, FOCUS was aimed to assess a new indication for a well-known antidepressant. It is almost impossible to believe that a doubleblind, placebo-controlled RCT could resemble real-world practice [2]. Any practicing physician can acknowledge that in usual care patients know-and agree with their physicians - what treatment will they receive. In real-world settings, it is not possible to mask patients and/or physicians in medicine RCTs [10]. Needless to say, it is almost impossible to imagine a situation in which patients will agree to be treated with a placebo. There is no doubt that in FOCUS, both participants and clinicians/investigators were placed in a situation that was very far from normal clinical practice. The explanations of the trial objectives, -a new indication with no regulatory approval granted-, procedures, -including the need for the use of placebo and that participants would have $50 \%$ chance of being treated with placebo-, and the informed consent process, were some of the features that prevent correctly tagging this trial as pragmatic. In the follow-up period, investigators checked the adherence to treatment by, among other measures, counting capsules when unused ones were returned: this is not a usual care. By doing this, investigators reported that adherence to treatment by trial participants was superior to that reported in routine clinical practice [9], a way to acknowledge that FOCUS procedures helped to separate the trial from normal care. Contrary to what happened with the Salford COPD trial, the term "pragmatic" was not mentioned in any document of the FOCUS trial: neither in the protocol [11] or statistical analysis [12] nor in the information posted on the European (EU-CTR 2011-005616-29) and British (ISRCTN83290762) registries, the latter last edited on October 15, 2018. It seems, therefore, that "pragmatic" was added into the article at the very last minute. The fact that the authors most likely wanted to attract the attention of potential readers by using the term "pragmatic" is supported by the fact that it was included in the article's title and abstract. But, interestingly enough, it was only mentioned twice in the article's text. FOCUS authors did not provide any explanation as to why this study should be considered pragmatic [9]. No other terms linked to pragmatic RCTs such as "real-world," "effectiveness," and "usual (normal) care or clinical practice" were used in the article.

Labeling as "pragmatic" pre-licensing trials-aiming to assess the efficacy/safety of investigational medicines or of new indications - is misleading. Journal editors should prevent this from happening during the editorial process. A reasonable approach is to limit the use of the adjective "pragmatic" to those RCTs that showed a high degree of pragmatism by means of the PRECIS-2 tool [1]. At the time of manuscript submission, editors should ask authors to submit, as supplemental information, the assessment of pragmatism by PRECIS- 2 tool (providing the scores of the nine domains); if the trial shows to be pragmatic, this assessment should be publicly disclosed to inform readers [2]. For those editors that do not want to engage in this type of exercise, not accepting as pragmatic any masked (participant, physician/ investigator) RCT is strongly suggested. By doing this, editors will educate authors (and readers) in the correct use of this scientific term that ultimately will help the accurate use of it by the clinical trials and regulatory communities.

Funding/support This work required no funding.

\section{Compliance with ethical standards}

Conflict of interest The author declares that he has no conflict of interest.

\section{References}

1. Loudon K, Treweek S, Sullivan F, Donnan P, Thorpe KE, Zwarenstein M (2015) The PRECIS-2 tool: designing trials that are fit for purpose. BMJ. 350:h2147

2. Dal-Ré R, Janiaud P, Ioannidis JPA (2018) Real-world evidence: how pragmatic are randomized controlled trials labeled as pragmatic? BMC Med 16:49

3. Janiaud P, Dal-Ré R, Ioannidis JPA (2018) Assessment of pragmatism in recently published randomized clinical trials. JAMA Intern Med 178:1278-1280

4. Vestbo J, Leather D, Bakerly ND, New J, Gibson JM, McCorkindale S et al (2016) Salford Lung Study Investigators. Effectiveness of fluticasone furoate-vilanterol for COPD in clinical practice. N Engl J Med 375:1253-1260

5. Dal-Ré R, Rosendaal F (2018) Efficacy and effectiveness: the wrong use of different terms. Eur J Intern Med 54:e17-e18

6. Dal-Ré R (2018) Could phase 3 medicine trials be tagged as pragmatic? A case study: the Salford COPD trial. J Eval Clin Pract 24: 258-261

7. Woodcock A (2017) Commentary: view from the frontline of pragmatic trials. BMJ 357:j2837

8. Pate A, Barrowman M, Webb D, Pimenta JM, Davis KJ, Williams $R$ et al (2018) Study investigating the generalisability of a COPD trial based in primary care (Salford Lung Study) and the presence of a Hawthorne effect. BMJ Open Resp Res 5:e000339

9. FOCUS Trial Collaboration (2019) Effects of fluoxetine on functional outcomes after acute stroke (FOCUS): a pragmatic, doubleblind, randomised, controlled trial. Lancet 393:265-274

10. Zwarenstein M, Treweek S, Gagnier JJ, Altman DG, Tunis S, Haynes B, Oxman AD, Moher D, for the CONSORT and Pragmatic Trials in Healthcare (Practihc) groups (2008) Improving the reporting of pragmatic trials: an extension of the CONSORT statement. BMJ. 337:a2390

11. Mead G, Hackett ML, Lundström E, Murray V, Hankey GJ, Dennis M (2015) The FOCUS, AFFINITY and EFFECTS trials studying the effect(s) of fluoxetine in patients with a recent stroke: a study protocol for three multicentre randomised controlled trials. Trials. $16: 369$

12. Graham C, Lewis S, Forbes J, Mead G, Hackett ML, Hankey GJ, Gommans J, Nguyen HT, Lundström E, Isaksson E, Näsman P, 
Rudberg AS, Dennis M (2017) The FOCUS, AFFINITY and EFFECTS trials studying the effect(s) of fluoxetine in patients with a recent stroke: statistical and health economic analysis plan for the trials and for the individual patient data meta-analysis. Trials. 18: 627
Publisher's Note Springer Nature remains neutral with regard to jurisdictional claims in published maps and institutional affiliations. 